\title{
Bibliografía citada y consultada
}

Anasb. Norme tecniche di selezione. Associazione Nazionale Allevatori Specie Bufalina. Caserta, s.d.

ABCZ. Arquivos da Associação Brasileira dos Criadores de Zebu. Consulta 13 de julio de 2010. Uberaba. MG.

Associação Brasileira dos Criadores de Zebu, ABCZ. Seminário Nacional. Revisão de critérios de seleção e julgamento em gado de corte. MG. 1998.

Alapati A, Kapa SR, Keepalyam S, Rangappa SMP, Yemireddy KR. Development of the body condition score system in Murrah buffaloes: validation through ultrasonic assessment of body fat reserves. $J$. Vet. Sci. (2010), 11(1), pp. 1-8. DOI: 10.4142/jvs.2010.11.1.1

Battacone, G. La valuatazione lineare dei bovini. R\&S Agraria. S. F., disponible en: http:/ / rsagraria.altervista. org/zootecnica\%20generale/valutazione_lineare.pd

B.I.F. Guidelines for uniform beef improvement programs. 2002. $8^{\text {th }}$ edition.

Binotti G. Evaluación fenotípica y mejoramiento genético del búfalo Mediterráneo italiano. En: Encuentro Nacional de Criadores de Búfalo. Bucaramanga. 2010.

Bergmann JAG. Indicadores de Precocidade Sexual em Bovinos de Corte. En: Congresso Brasileiro das Raças Zebuínas. Uberaba: ABCZ. 1998, pp. 145-155.

Bonsma JC. System of judging for functional. En: Factors Affecting Calf Crop. University of Florida Press. $3^{\text {a }}$. ed., 1973.

. Judging cattle for functional efficiency. Brahman Journal. Pretoria. South Africa, nov. 1993, pp. 15-24.

Costa CN, Josahkian LA, Machado CHC. A evolução do Serviço de Controle Leiteiro da ABCZ na década de 90. Informativo ABCZ. feb.-mar., 2000, 16 (150), p. 13.

Daly JJ. Breeding for beef production. Queensland Department of Primary Industries, 1977.

Dickerson G. Implications of genetic-environmental interactions in animal breeding. Anim Prod, 1962, 4, pp. 47-63.

Eler JP, Ferraz JBS, Silva PR. Parâmetros genéticos de escores visuais e circunferência escrotal em bovinos da raça Nelore. En: Reunião anual da sociedade brasileira de zootecnia, XXXII, Brasília DF. Brasil, 1995, pp. 739-740.

Falconer DS, Mackay TF. Introducción a la genética cuantitativa. Zaragoza. Editorial Acribia, 2001.

Fries LA, Albuquerque LG. Julgamento de raças zebuínas: Genética e Melhoramento Animal. Uberaba. ABCZ/FAZU, 1999. (Módulo IV).

Fries LA. Anexo conceitual sobre as três precocidades. En: III Simpósio o nelore do século XXI. Ribeirão Preto, Ribeirão Preto. SP. ACNB, 1995, pp. 56-61.

Uso de escores visuais em programas de seleção para a produtividade em gado de corte. En: Seminário nacional - revisão de critérios de julgamento e seleção em gado de corte, Uberaba. MG. 1996, pp. 39-44.

Guitou H, Monti A. Interpretación y uso correcto de los DEP como herramienta de selección. Programa de examen. Unidad de genética animal. Inta. Castelar. Argentina, 1998.

Gutiérrez M. Buffalo production perspectives in Colombia. En: Proceedings VI World Buffalo Congress: an alternative for animal agriculture in the third millenium. Maracaibo, Venezuela. 2001, pp 677-697.

Icar. Guidelines for buffalo milk recording for low to medium and medium to high input production systems. 2002, disponible en: http://www.icar.org/pages/recording_guidelines.htm 
Icar. Annex 1. Working documents. En: Workshop for improvement breeding and management strategies for buffaloes Bled, Slovenia. 2000, pp. 13-16.

Josahkian LA. Avaliação zootécnica e funcional. ABCZ. 2007.

. Métodos e critérios de julgamento. En: Curso de Noções de Morfologia e Julgamento de Zebuínos - ABCZ, Uberaba, MG. 2010. http://www.abcz.org.br

Josahkian LA, Machado CHC, Koury FW. Manual do programa de melhoramento genético das raças zebuínas. Uberaba: ABCZ, 2003. 1998.

. Manual do programa de melhoramento genético das raças zebuínas. Uberaba: ABCZ,

Kearsey MJ, Pooni HS. Genetic analysis of quantitative traits. Londres, Chapman and Hall, 1996.

Koury FW. Análise genética de escores visuais e suas respectivas relações com desempenho ponderal na raça Nelore. Pirassununga, Dissertação de Mestrado. SP. USP. 2001.

Koury FW, Ferraz JBS, Eler JP, Meister NC, Pineda NR. Estimativas de herdabilidades e correlações genéticas entre escores de avaliações visuais e características de desenvolvimento ponderal em uma população da raça Nelore. En: IV Simposio nacional de melhoramiento animal. Campo Grande. MS. 2002.

Lasley JF. Genética del mejoramiento del ganado, sistemas de cruzamiento y selección de ganado vacuno lechero. México. Uthea. 1979.

Lima FP, Bonilha LM, Razook AG, Pacola LJ, Figueredo LA, Peixoto AM. Parâmetros genéticos em características morfológicas em bovinos Nelore. Bol. Indust. Animal. 1989. 46 (2), pp. 249-257.

Lynch M, Walsh B. Genetics and analysis of quantitative traits. Massachusetts. Sinauer Associates. 1998.

Machado CHC. "Exterior e anatomia de zebuínos", En: Curso de especialização de julgamento de zebuinos. Apostila. Uberaba, FAZU. 2003.

Ministério da Agricultura. Estatutos e Regulamentos do Serviço de Registro Genealógico - A.B.C.B. Padrões Raciais. Brasília, DF, 1976.

Mrode RA. Linear models for the prediction of animal breeding values. CAB International. Wilts, UK. 1996.

Mullick DN. Review of the investigations on the physiology of Indian buffaloes. J. Dairy Sci. 1964, 17, pp. $45-50$.

Peixoto, A.M. Exterior e julgamento de bovinos, ESALQ-CAS, 1968.

Pineda NR. Influência do Zebu na produção de carne no Brasil. In: Simpósio Nacional de Melhoramento Animal. Belo Horizonte, SBMA. 3, 2000.

Ramos AA. Curso de juzgamento de bubalinos (exterior, tipo y razas). Asociación Colombiana de Bufalistas - ACB, Córdoba, Colombia, 2008.

. Exterior, raças e julgamento de bubalinos para leite e carne. Apontamentos de aula. 2000.

Melhoramento genético de bubalinos. En: III Simpósio de búfalos de las Américas y 2nd buffalo symposium of the Europe and Americas. Memorias. Medellín, Colombia, 2006.

. Taller de tipo, raza y juzgamiento de bubalinos para producción de carne y leche. En: IV Simposio de búfalos de las Américas. Mérida, Venezuela, 2008.

Rocha CE. Estudo de fatores que podem influenciar o valor da carcaça em bovinos da raça Nelore. UNESP, Jaboticabal. SP. Dissertação de Mestrado. 1999.

Rossi. Estado corporal de las vacas adultas. En: Difusión Ganadera, la cría del siglo XXI. 1999. Reproducido en Veterinaria Argentina, 16 (159), pp. 677-683. Disponible en: VetUy Agro y Veterinaria. http:// www.vet.uy.com/articulos/bovinos/100/0078/bov078.htm 
Sampaio N de S. Exterior de zebuínos. En: Curso intensivo de julgamento de zebuínos. 26-30 jul. Curso. 41 Uberaba: ABCZ, 1999, s.p.

Scarpati MTV, Magnabosco CU, Josahkian LA. Estudos de medidas corporais e peso vivo em animais jovens da raça Nelore. En: Reunião anual da sociedade brasileira de zootecnia, 33. Fortaleza. CE. Brasil. SBZ, 1996. v. 1, pp. 110-112.

Trovo J B de F, Razook AG. Fundamentos da avaliação genética. En: Curso sobre Avaliação Genética de Bovinos de Corte em Goiás. Anais, Goiânia: NRG/ EV/UFG, 25-27 sep. 1996.

Warwick EJ, Legates J. Cría y mejora del ganado. 3ª ed. México, Mcgraw-Hill, 1980.

Se terminó de imprimir en junio de 2001 en los talleres gráficos de

Editorial Artes y Letras S.A.S. 


\section{Apoyo}

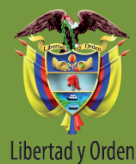

Ministerio de Agricultura y Desarrollo Rural

República de Colombia

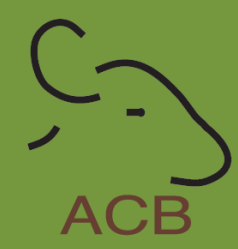

Asociación Colombiana de Criadores de Búfalos

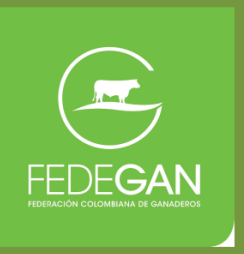

